\title{
Can the Rectum Balloon Minimize Rectal Toxicity During Irradiation Therapy of Prostate Cancer?
}

\author{
Nuria Landwehr, Manfred Georg Krukemeyer, Wolfgang Wagner \\ Paracelsus-Klinik Osnabrück, Osnabrück, Germany \\ Email: prof.wolfgang.wagner@paracelsus-kliniken.de
}

Received December 6, 2012; revised January 16, 2013; accepted January 24, 2013

\begin{abstract}
The irradiation of the rectum cancer occurs in many institutes by using a rectum balloon in order to reduce the mobility of the target organ and to distance the rectum from the target organ. The objective is to reduce side effects quantitatively as well as qualitatively. On the basis of two hospitals using identical techniques for the prostate irradiation with the sole difference of the rectum balloon the toxicity has been evaluated with the result of no significant difference between the cohorts concerning diarrhea, rectal pain symptoms and rectal bleedings. Therefore the authors consider the use of the rectum balloon prior to each irradiation not necessary for the reduction of toxicity particularly due to the fact that the application is often very painful, especially for patients with hemorrhoids. The rectum balloon as a tool for the reduction of the prostate mobility was not objective of this study.
\end{abstract}

Keywords: Prostate Cancer; Radiotherapy; Rectum Balloon; Side Effects

\section{Introduction}

Irradiation today is one of the main therapy modalities in patients with prostate cancer and can be used in all stages with similar results in comparison with surgery. Being aware of the physical occurrences such as the dose-response-relationship it is the objective to achieve the optimal dosage for the tumor control taken into consideration the toxicities for the neighboring organs. Modern therapy modalities like 3D conformal irradiation techniques or IMRT are able to realize dose escalation and minimize side effects. However also supplementary techniques for the reduction of rectal and urogenital side effects are desirable. This is the original idea for the use of the intrarectal balloon for the rectal protection. On the one hand the rectal balloon could facilitate a reproducible prostate localization which is fundamental for the exact irradiation planning. On the other hand the intrarectal balloon distances with its voluminous structure especially the dorsal circumference out of the irradiation area and therefore minimizes at least theoretically the rectal toxicity.

\section{Material and Methods}

Two patients cohorts in two hospitals in northern Germany (clinic A and clinic B, each with 51 patients) have been evaluated retrospectively concerning general condition (WHO score), age, TNM stage, gleason score and PSA value. On average there was a WHO score between
0 - 1 corresponding to a good/satisfactory general condition, the age of the patients was in the median in clinic A approx. 65 years and in clinic B approx. 69 years, a TNM stage of $\leq$ T2N0M0 (variance T1a N0 M0 G1 - T4 N0 M0) and a gleason score of 6 (variance 3 - 9) and a PSA value of 16.7 (clinic A) resp. 17.1 (clinic B) (variance 1.7 $>40$ ). Both cohorts consisted of patients previously not operated so that biopsies had been performed for a histological data examination. These patients should be treated in curative intention by means of a definitive radiotherapy. The radiotherapy was planned 3D-conformally and checked regulary for correctness on the basis of verification imaging. Both groups were irradiated with $3 \mathrm{D}$ conformal treatment with a total dose of 70.2 Gy resp. 71.2 Gy using a fraction dose of 1.8 to $2.0 \mathrm{~Gy}$. The patients in clinic $\mathrm{A}$ had been irradiated using daily a rectum balloon filled with $30 \mathrm{ml}$ of air. The rectum contouring occurred in clinic $\mathrm{A}$ on the basis of the rectum balloon and in clinic $\mathrm{B}$ the radiation therapist orientated on the rectum exterior wall. It is well-known that filling-caused modifications of the rectum volumes cannot be avoided. The well-known TD5/5 limit values were respected on the basis of the individual dosis-volume histograms. In clinic A 18 patients were treated with a medicinal hormone withdrawal therapy and in clinic B 40 patients. 3 patients were subjected to a surgical castration in the sense of orchiectomy. The difference was due to the fact that in clinic B all patients of the intermediary risk group have been treated with a (neoadjuvant, 6-month) hormone 
ablative therapy whereas in clinic A the patients of the high risk group have primarily been treated with a longterm hormone withdrawal therapy as recommended in the guidelines. Up to a dose of approx. 50 Gy the seminal vesicles have been irradiated too. In terms of a retrospective study modality the required data collection for the statistic evaluation occurred on the basis of the irradiation protocols as well as examinations during and after the irradiation therapy in the further follow-up. The irradiation-induced side effects were evaluated and listed according to RTOG/EORTC criteria. Consequently it has been divided into acutely occurring side effects within irradiation and subacutely up to 3 months after the irradiation end prevailing complaints as well as up to 24 months postactinically occurring side effects. The statistical data collection occurred in both clinics by means of a junction of the patient files in Microsoft Excel tables. The approximative normal test is used as statistic test.

\section{Results}

\section{Rectal Side Effects}

In the patient base of clinic $\mathrm{A}$ in total 18 patients complained of $1^{\text {st }}$ grade side effects (proctitis/minor bleeding/diarrhea $<5 \times$ per day) and of $2^{\text {nd }}$ grade 4 patients (colitis/stronger bleedings requiring treatment/diarrhea $>$ $5 \times$ per day).

Patients with pains only during the rectum balloon application have not been considered.

Comparably in the patient base of clinic B in 20 patients have been noticed $1^{\text {st }}$ grade toxicities and in 2 patients $2^{\text {nd }}$ grade toxicities.

Although in the total amount the rectal clinical toxicities are comparable, a remarkable difference has been evaluated at the more detailed examination of the rectal pains and bleedings, especially in their chronological development (Figure 1).

A statistically significant difference in the $95 \%$-confidence interval (approximative normal test) has not been achieved, however the difference to be demonstrated was obvious.

Especially by means of the differentiated and temporal sorted listing the trend to the often complained acute to subacute pains has become evident (Figure 2).

To avoid that an existing hemarrhoidal suffer determines this result, these patients have been evaluated separately. Therefore it becomes apparent that the patients with hemarrhoidal pains have been comparably in both cohorts (clinic A 90\%, clinic B 100\%).

A homolog development has been evaluated in case of the rectal bleedings:

In both cohorts perianal bleedings have been noticed similarly often, $\mathrm{n}=8(15.7 \%)$. In most cases temporally

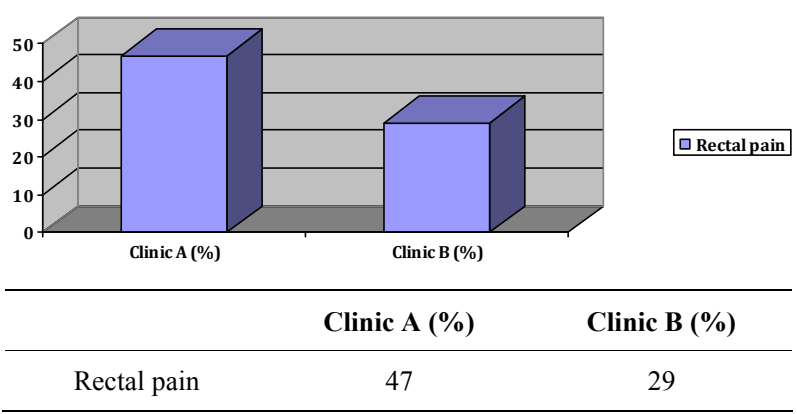

Figure 1. Rectal pain.

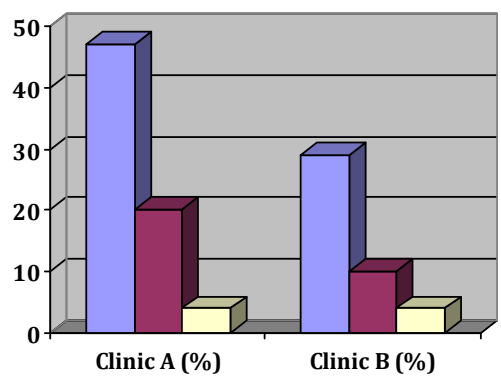

$\square$ Acute rectal pain

$\square$ Subacute rectal pain

$\square$ Chronic rectal pain

Figure 2. Acute/subacute/chronic rectal pain.

limited minor bleedings have been occurred.

In the patient base of clinic A have occurred tendentially more acute than subacute or later bleedings, although not in a statistically significant dimension. Especially in patients with hemorrhoids in the cohort with the rectum balloon have been evaluated acute rectal bleedings (Figures 3(a) and (b)).

A diarrhea according to RTOG $1^{\circ}-2^{\circ}$ grade has occurred in the patient base with the application of the intrarectal balloon in 10 patients, a diarrhea $3^{\circ}$ grade in 2 patients, in total therefore approximatively $24 \%$ of the patients.

In the cohorts without application of the rectum balloon a diarrhea $1^{\circ}-2^{\circ}$ grade has occurred in 11 patients, corresponding approximatively to $22 \%$ of the patients (Figure 4).

The result for the general rectal toxicity is summarized (Figure 5).

In the acute to subacute phase we have evaluated a minor difference out of the $95 \%$ confidence interval. During the later process both collectives have aligned to each other.

Comparison of the occurring side effects-Overview (Figure 6).

\section{Summary}

In both patients bases side effects have occurred comparably often, however with the difference that the patients irradiated with the rectum balloon application in total, especially in the acute to subacute phase more often suffer from rectal pains independently from a hemorrhoidal 


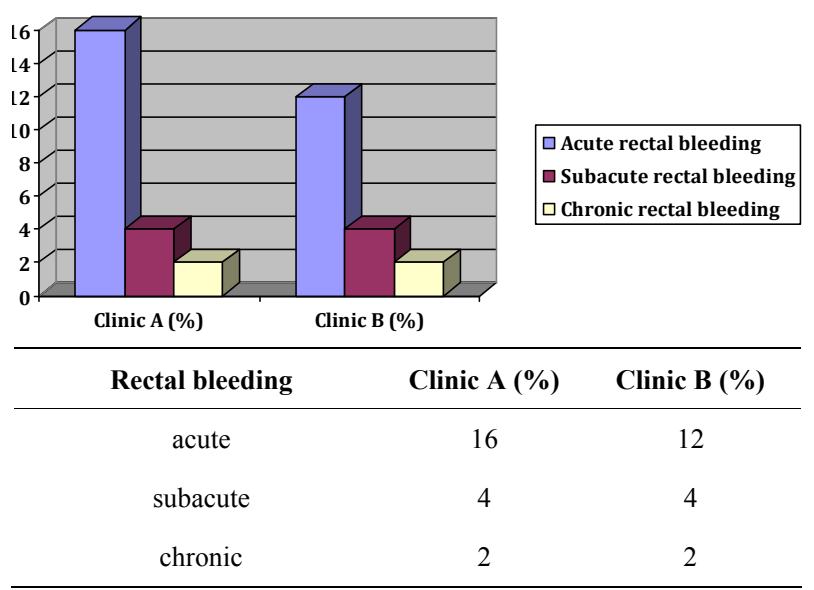

(a)

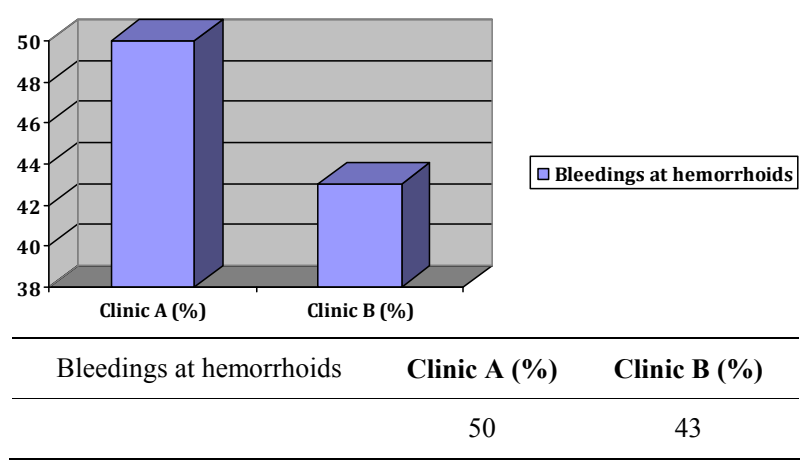

(b)

Figure 3. (a) Acute/subacute/chronic rectal bleeding; (b) Bleedings at hemorrhoids.

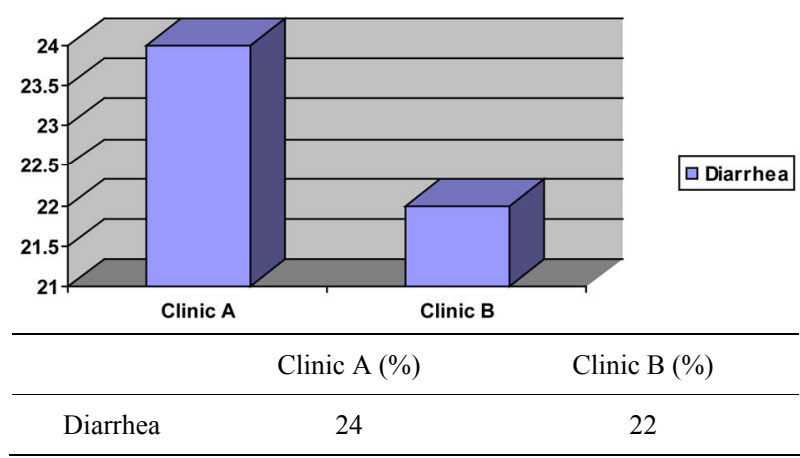

Figure 4. Diarrhea.

complaint. These patients have demonstrated increased acute rectal bleedings, especially those with preexisting hemorrhoids.

Although a statistically significant result in the $95 \%$ confidence interval has been narrowly missed, a remarkably trend cannot be ignored that use of the rectum balloon here causally comes into question. The condition that the cohorts have aligned to each other in the later process regarding the general rectal toxicity is reinforcing this hypothesis.

A protective influence regarding the radiation-induced

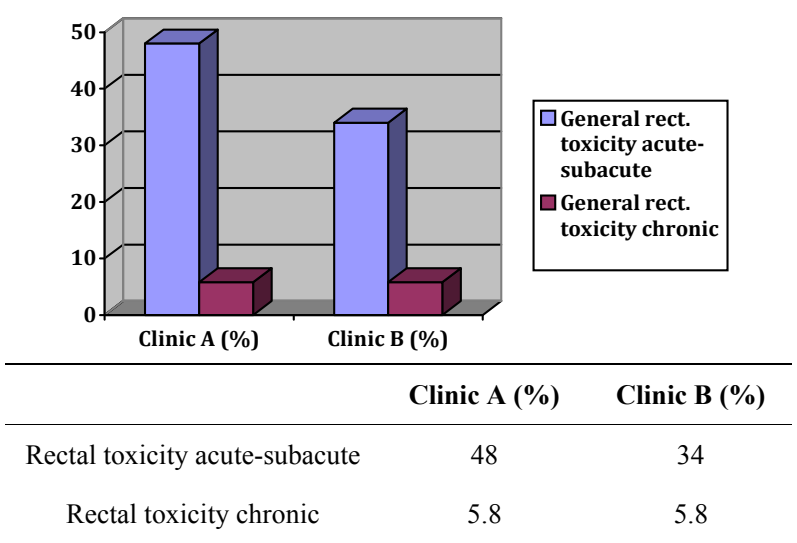

Figure 5. General rectal toxicity.

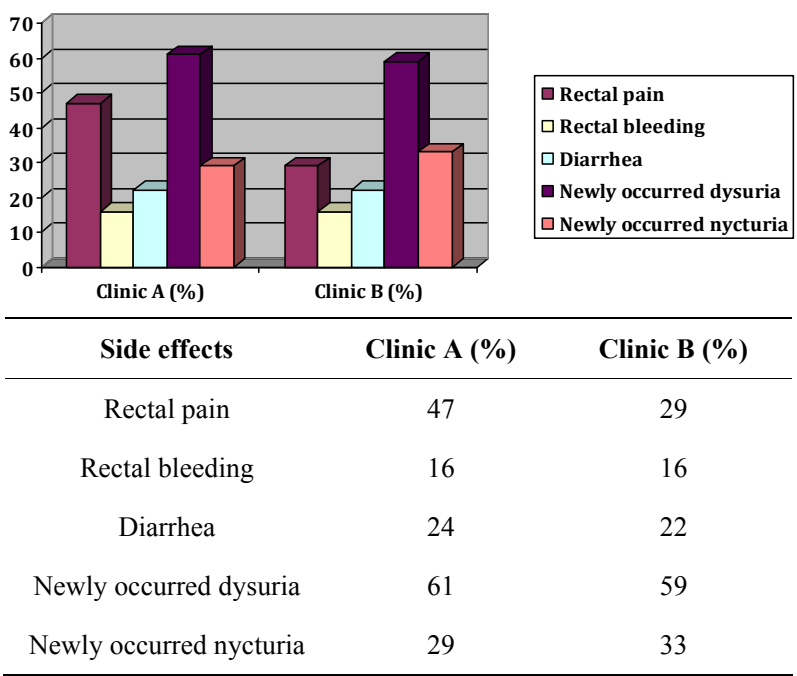

Figure 6. Overview of side effects.

side effects by means of the rectum balloon has not been verified with this study.

\section{Discussion}

The challenge of the radiotherapy is to obtain the optimal dose in the target volume considering the toxicity for the adjacent structures. The knowledge of the dose-effect relationship has been established in numerous studies [1-7]. The dose escalation by means of most modern methods as the $3 \mathrm{D}$ conformal irradiation is an acknowledged effective strategy in the therapy of the organ defined prostate carcinoma.

The effectivity of a higher irradiation dose is relevant especially for patients with an intermediate and high risk profile $[5,6,8]$. The biochemical cure rates seem to be roughly comparable at least for the stages T1-T2 between conformation irradiation (min. 72 Gy), the permanent Seed implantation, the combined brachy and external radiotherapy as well as the radical prostatectomy [9-11]. However consecutive higher irradiation-induced rectal 
and urogenital side effect rates, which are subsumed under the term volume effect, are also well documented [12-17].

A higher irradiation dose leads to an increasing incidence of acute and later side effects. Especially the cumulative risk for later gastrointestinal side effects of $2^{\circ}$ grade and higher according to RTOG criteria rises significantly [17]. If dose-volume-histograms are considered for the evaluation of the resulting toxicities for the neighboring organs with optimal configuration of the target volume to be irradiated, an exponential rise of complications can be noticed by using an increased irradiation dose $[14,15,18,19]$. The percentage of the irradiated volume is primarily the decisive factor for the toxicity, less the absolute volume [14].

To configurate the irradiation area as accurate as possible the intrarectal balloon promises at the irradiation of the prostate carcinoma to be a successful approach under the following conditions:

The reproducibility of the prostate localization is a practical-technical problem which complicates the smallvolume irradiation planning. Rectum protection proponents believe that the rectum balloon reduces especially the interfractional prostate shift [20-24]. By contrast the intrafractional shift dimensions are minor than the interfractionals [25], however with increasing rectum volume appear to be larger. This would argue against the use of the rectum balloon. The natural different rectal gas and stool quantity as influencing factor is beyond the radiotherapist's knowledge [26]. Some studies suggest that the interfractional shifts of the prostate are reduced only in statistically insignificant dimension, especially in the anterior-posterior direction [27-30]. Since in the comparative group a rectum balloon has not been applied, an evaluation considering this aspect was not possible and therefore it is not the object of this study.

The posterior and partially lateral component of the rectal wall is distanced out of the irradiation area by means of the voluminous structure of the rectum balloon equally at the $3 \mathrm{D}$-conformal irradiation or the IMRTtechnique or the stereotaxis [26,30,31]. Considering the dose-volume histograms the intrarectal balloon seems to reduce the rectum dose [26,30-35]. The application of the rectum balloon leads to significant dose-volume-reducing results for the rectum, partially illustrated by film dosimetry.

The interpretation of rectal dose-volume histrograms critically examined states a problem. So the different contouring of the rectum at a missing consent for the determination of the rectal limit $[2,11,36]$ seems to determine the missing reproducibility of the results with a high inter- and intra-individual variability of the DVH parameters.

Therefore the values of the relative and absolute vol- ume fraction vary a lot for each patient depending already on the selection of the top and bottom rectum limits $[36,37]$.

Besides studies show that by means of the intrarectal balloon a dose reduction at the posterior rectum wall is opposed to a higher dose fraction at the anterior rectum wall [38].

The present study examines the clinically actually occurring intra and postactinic side effects.

It becomes apparent that in both cohorts in their entirety of the documented toxicities (predominantly $1^{\circ}-2^{\circ}$ grade) a significant difference could not be detected. It became clear that in contrast to the physical benefit supposed on closer examination of the dose-volume-histograms, that based on the up to 24 months post-actinically documented clinic side effects this benefit could not be verified.

Furthermore with the application of the rectum balloon more acute bleedings occur (12\% versus $16 \%)$, especially in case of hemorrhoidal complaints. The situation is similar with rectal pains which obviously leads more often in the group with the rectal balloon to (sub) acute complaints in terms of a proctitis (29\% versus $47 \%$ ). Here, the statistic significance in the $95 \%$-confidence interval has been narrowly missed (approximative normal test). There was no difference between the cohorts regarding more frequent defecation up to discrete fecal incontinence. This is not astonishing since the pathophysiology considers especially the irradiation of the anal canal and the inferior rectum to be the cause of rectal incontinence [3]. Therefore the protective effect of the rectum balloon regarding rectal continence seems to be questionable anyway.

In the late follow-up (24 months examination) both cohorts were converging and have demonstrated a comparable rectal toxicity $(6 \%$ versus $6 \%$ ). Based on this clinical study there appear to be grounds for suspicion that the different trend in the above mentioned items in the intra and acute postactinic phase is resulting from a mechanical irritation due to the daily application of the rectum balloon. The fundamental idea of the rectum bal loon with dose-volume calculations is a concept to be taken into account considering economical aspects; future-oriented therapy options such as the combination of percutaneous and interstitial radiation and the wider use of IMRT could generate unquestionably a reasonable advantage for the patients concerning the reduction of radiation-induced toxicities $[8,39,40]$.

\section{Conclusions}

Although numerous studies at evaluation of dose-volumehistograms seem to show a dose-volume-reducing effect by applying the intrarectal balloon for the rectum, the correct interpretation of dose-volume histograms seems 
to be difficult due to the above mentioned circumstances and its importance obviously relativizes against the background of the actually occurring side effects. Finally arises the question if the rectum balloon irritates additionally the rectal mucosa which is already affected by the irradiation in a way that a possible protective effect is at least eliminated. According to the present study dosimetric calculations clinically cannot withstand to their predictive value. Within this context the detrimental impact on the patient's quality of life by means of the daily rectum balloon application would not appear to be justified.

However the evaluation of the balloon significance regarding the immobilisation of the target organ has not been the aim of this research.

\section{REFERENCES}

[1] G. E. Hanks, K. I. Martz and J. J. Diamond, "The Effect of Dose on Local Control of Prostate Cancer," International Journal of Radiation Oncology, Biology, Physics, Vol. 15, No. 6, 1988, pp. 1299-1305. doi:10.1016/0360-3016(88)90224-6

[2] G. E. Hanks, T. E. Schultheiss, M. Hunt and B. Epstein, "Factors Influencing Incidence of Acute Grade 2 Morbidity in Conformal and Standard Radiation Treatment of Prostate Cancer," International Journal of Radiation Oncology, Biology, Physics, Vol. 31, No. 1, 1995, pp. 25-29. doi:10.1016/0360-3016(94)00366-S

[3] G. E. Hanks, A. L. Hanlon, W. Pinover, E. Horwitz and T. Schultheiss, "Dose Selection for Prostate Cancer Patients Based on Dose Comparison and Dose Response Studies," International Journal of Radiation Oncology, Biology, Physics, Vol. 57, No. 2, 2000, pp. 384-390.

[4] S. Levegrün, A. Jackson, M. Zelefsky, et al., "Analysis of Biopsy Outcome after Three-Dimensional Conformal Radiation Therapy of Prostate Cancer Using Dose-Distribution Variables and Tumor Control Probability Models," International Journal of Radiation Oncology, Biology, Physics, Vol. 47, No. 5, 2000, pp. 1245-1260. doi:10.1016/S0360-3016(00)00572-1

[5] A. Pollack, G. K. Zagars, L. G. Smith, H. Lee, et al., "Preliminary Results of a Randomized Radiotherapy, Dose-Escalation Study Comparing 70 Gy with 78 Gy for Prostate Cancer," Journal of Clinical Oncology, Vol. 18, No. 23, 2000, pp. 3904-3911.

[6] Z. Symon, K. A. Griffith, P. W. Laughlin, et al., "Dose Escalation for Localized Prostate Cancer: Substantial Benefit Observed with 3-D-Conformal Therapy," International Journal of Radiation Oncology, Biology, Physics, Vol. 57, No. 2, 2003, pp. 384-390. doi:10.1016/S0360-3016(03)00569-8

[7] R. Valicenti, J. Lu, M. Pipepich and D. Grignon, "Survival Advantage from Higher-Dose Radiation Therapy for Clinically Localized Prostate Cancer Treated on the Radiation Therapy Oncology Group Trials," Journal of Clinical Oncology, Vol. 18, No. 14, 2000, pp. 2740-2746.

[8] M. J. Zelefsky, Z. Fuks, M. Hunt, et al, "High-Dose In- tensity Modulated Radiation Therapy for Prostate Cancer: Early Toxicity and Biochemical Outcome in $722 \mathrm{~Pa}-$ tients," International Journal of Radiation Oncology, Biology, Physics, Vol. 53, No. 5, 2002, pp. 1111-1116. doi:10.1016/S0360-3016(02)02857-2

[9] P. A. Kupelian, L. Potters, D. Khuntia, et al., "Radical Prostatectomy, External Beam Radiotherapy $<72$ Gy, External Beam Radiotherapy $<72$ Gy, Permanent Seed Implantation or Combined Seeds/External Beam Radiotherapy for Stage T1-T2 Prostate Cancer," International Journal of Radiation Oncology, Biology, Physics, Vol. 58, No. 1, 2004, pp. 25-33. doi:10.1016/S0360-3016(03)00784-3

[10] P. A. Kupelian, M. Elshaikh, C. Reddy, C. Zippe and E. A. Klein, "Comparison of the Efficacy of Local Therapies for Localized Prostate Cancer in the Prostate-Specific Antigen Era: A Large Single-Institution Experience with Radical Prostatectomy and External-Beam Radiotherapy," Journal of Clinical Oncology, Vol. 20, No. 16, 2002, pp. 3376-3385. doi:10.1200/JCO.2002.01.150

[11] L. Potters, E. A. Klein, M. W. Kattan, P. A. Kupelian, et al., "Monotherapy for Stage T1-T2 Prostate Cancer: Radiacal Prostatectomy, External Beam Radiotherapy or Permanent Seed Implantation," Radiotherapy \& Oncology, Vol. 71, No. 1, 2004, pp. 29-33. doi:10.1016/j.radonc.2003.12.011

[12] D. B. Chism, E. Horwitz, A. Hanlon, W. Pinover, et al., "Late Morbidity Profile in Prostate Cancer Patients Treated to 79-84 Gy by a Simple Four-Field Coplanar Beam Arrangement," International Journal of Radiation Oncology, Biology, Physics, Vol. 55, No. 1, 2003, pp. 71-77. doi:10.1016/S0360-3016(02)03822-1

[13] C. Fiorino, C. Cozzarini, V. Vavassori, G. Sanguinetti, et al., "Relationships between DVHs and Late Rectal Bleeding after Radiotherapy for Prostate Cancer: Analysis of a Large Group of Patients Pooled from Three Institutions," Radiotherapy \& Oncology, 64, No. 1, 2002, pp. 1-12. doi:10.1016/S0167-8140(02)00147-0

[14] E. H. Huang, A. Pollack, L. Levy, et al., "Late Rectal Toxicity: Dose-Volume Effects of Conformal Radiotherapy for Prostate Cancer," International Journal of Radiation Oncology, Biology, Physics, Vol. 54, No. 5, 2002, pp. 1314-1321. doi:10.1016/S0360-3016(02)03742-2

[15] A. Jackson, M. W. Skwarchuk, M. Zelefsky, et al., "Late Rectal Bleeding after Conformal Radiotherapy of Prostate Cancer (II): Volume Effects and Dose-Volume Histograms," International Journal of Radiation Oncology, Biology, Physics, Vol. 49, No. 3, 2001, pp. 685-698. doi:10.1016/S0360-3016(00)01414-0

[16] J. M. Michalski, J. A. Purdy, K. Winter, M. Roch III, et al., "Preliminary Report of Toxicity Following 3-D-Radiation Therapy for Prostate Cancer on 3 DOG/RTOG 9406," International Journal of Radiation Oncology, Biology, Physics, 46, No. 2, 2000, pp. 391-402. doi:10.1016/S0360-3016(99)00443-5

[17] S. T. Peeters, W. Heemsbergen, W. L. J. Putten, et al., "Acute and Late Complications after Radiotherapy for Prostate Cancer: Results of a Multicenter Randomized Trial Comparing 68 Gy to 78 Gy," International Journal of Radiation Oncology, Biology, Physics, Vol. 61, No. 4, 2005, pp. 1019-1034. doi:10.1016/j.ijrobp.2004.07.715 
[18] G. E. Hanks, Al. Hanlon, B. Epstein and E. Horwitz, "Dose Response in Prostate Cancer with 8 - 12 Years' Follow-Up," International Journal of Radiation Oncology, Biology, Physics, Vol. 54, No. 2, 2002, pp. 427-435. doi:10.1016/S0360-3016(02)02954-1

[19] G. J. Kutcher and C. Burman, "Calculation of Complication Probability Factors for Non-Uniform Normal Tissue Irradiation: The Effective Volume Method," International Journal of Radiation Oncology, Biology, Physics, Vol. 16, No. 6, 1989, pp. 1623-1630. doi:10.1016/0360-3016(89)90972-3

[20] A. V. D'Amico, J. Manola, M. Loffredo, et al., "A Practical Method to Achieve Prostate Gland Immobilization and Target Verification for Daily Treatment," International Journal of Radiation Oncology, Biology, Physics, Vol. 51, No. 5, 2001, pp. 1431-1436. doi:10.1016/S0360-3016(01)02663-3

[21] N. Gerstner, St. Wächter, D. Dörner, et al., "A Rectal Balloon Catheter as Internal Immobilization Device for Conformal Radiotherapy of Prostate Cancer," Strahlentherapie und Onkologie, 175, No. 5, 1999, pp. 232-238. doi:10.1007/BF02742401

[22] R. J. Smeenk, R. J. Louwe, K. M. Langen, et al., “An Endorectal Balloon Reduces Intrafraction Prostate Motion During Radiotherapy," International Journal of Radiation Oncology, Biology, Physics, Vol. 83, No. 2, 2012, pp. 661-669.

[23] B. S. Teh, W. Y. Mai, B. Uhl, et al., "Intensity-Modulated Radiation Therapy (IMRT) for Prostate Cancer with the Use of a Rectal Balloon for Prostate Immobilization: Acute Toxicity and Dose-Volume Analysis," International Journal of Radiation Oncology, Biology, Physics, Vol. 49, No. 3, 2001, pp. 705-712. doi:10.1016/S0360-3016(00)01428-0

[24] S. Wachter, N. Gerstner, K. Dieckmann, et al., "Three Dimensional Planned Conformal Low Volume Radiotherapy of Localized Carcinoma of the Prostate," Strahlentherapie und Onkologie, Vol. 173, No. 5, 1997, pp. 253260. doi:10.1007/BF03039434

[25] M. J. Ghilezan, A. D. Jaffray, J. H. Siewerdsen, et al., "Prostate Gland Motion Assessed with Cine-Magnetic Resonance Imaging (Cine-MRJ)," International Journal of Radiation Oncology, Biology, Physics, Vol. 62, No. 2, 2005, pp. 406-417. doi:10.1016/j.ijrobp.2003.10.017

[26] E. Van Lin, L. P. van der Vight, et al., "The Effect of an Endorectal Balloon and Off-Line Correction on the Interfraction Systematic and Random Prostate Position Variations: A Comparative Study," International Journal of Radiation Oncology, Biology, Physics, Vol. 61, No. 1, 2005, pp. 278-288. doi:10.1016/j.ijrobp.2004.09.042

[27] A. Y. Hung, M. Garzotto and D. Kaurin, "Minimal Benefit of an Endorectal Balloon for Prostate Immobilization as Verified by Daily Localization," Medical Dosimetry, Vol. 36, No. 2, pp. 195-199. doi:10.1016/j.meddos.2010.03.003

[28] C. W. Wang, F. C. Chong, M. K. Lai, Y. S. Pu, et al., "Set-Up Errors Due to Endorectal Balloon Positioning in Intensity Modulated Radiation Therapy for Prostate Cancer," Radiotherapy \& Oncology, Vol. 84, No. 2, 2007, pp.

\section{7-184. doi:10.1016/j.radonc.2007.06.009}

[29] S. Both, K. K. Wang, J. P. Plastaras, et al., "Real-Time Study of Prostate Intrafraction Motion during External Beam Radiotherapy with Daily Endorectal Balloon," International Journal of Radiation Oncology, Biology, Physics, Vol. 81, No. 5, 2011, pp. 1302-1309.

[30] B. S. Teh, L. Dong, J. E. Me Gary, et al., "Rectal Wall Sparing by Dosimetric Effect of Rectal Balloon Used during Intensity-Modulated Radiation Therapy (IMRT) for Prostate Cancer," Medical Dosimetry, Vol. 30, No. 1, 2005, pp. 25-30. doi:10.1016/j.meddos.2004.10.005

[31] K. Takayama, T. Mizowaki, Y. Negoro, et al., "Impact of Double-Balloon Rectal Catheter Use in External-Beam Radiotherapy for Prostate Cancer," International Journal of Clinical Oncology, Vol. 16, No. 1, 2011, pp. 50-56. doi:10.1007/s10147-010-0129-7

[32] A. L. Hartford, A. Niemierko, J. A. Adams, M. M. Urie, W. Shipley, et al., "Conformal Irradiation of the Prostate Estimating Long-Term Rectal Bleeding Risk Using DoseVolume Histograms," International Journal of Radiation Oncology, Biology, Physics, Vol. 36, No. 3, 1996, pp. 721-730. doi:10.1016/S0360-3016(96)00366-5

[33] R. Patel, N. Orton, W. A. Tome, et al., "Rectal Dose Sparing with Balloon Catheter and Ultrasound Localization in Conformal Radiation Therapy for Prostate Cancer," Radiotherapy \& Oncology, Vol. 67, No. 3, 2003, pp. 285-294. doi:10.1016/S0167-8140(03)00056-2

[34] M. V. Sanghani, J. Ching, D. Schultz, et al., "Impact on Rectal Dose from the Use of a Prostate Immobilization and Rectal Localization Device for Patients Receiving Dose Escalated 3-D-Conformal Radiation Therapy," Urologic Oncology, Vol. 22, No. 3, 2004, pp. 165-168. doi:10.1016/j.urolonc.2004.02.002

[35] R. J. Smeenk, E. N. van Lin, P. van Kollenburg, et al., "Endorectal Balloon Reduces Anorectal Doses in PostProstatectomy Intensity-Modulated Radiotherapy," Radiotherapy \& Oncology, Vol. 101, No. 3, 2011, pp. 465470. doi:10.1016/j.radonc.2011.07.019

[36] H. Geinitz, F. B. Zimmermann, L. Narkwong, et al., "Prostate Cancer: Difficulties Regarding the Interpretation of Rectal Dose-Volume Histograms," Strahlentherapie und Onkologie, Vol. 176, No. 4, 2000, pp. 168-172. doi:10.1007/s000660050052

[37] L. J. Boersma, M. van den Brin, A. M. Bruce, T. Shouman, et al., "Estimation of the Incidence of Late Bladder and Rectum Complications after High-Dose (70-78 Gy) Conformal Radiotherapy for Prostate Cancer Using DoseVolume Histograms," International Journal of Radiation Oncology, Biology, Physics, Vol. 41, No. 1, 1998, pp. 83-92. doi:10.1016/S0360-3016(98)00037-6

[38] H. Elshaved, T. Bölling, C. Moustakis, et al., "Organ Movements and Dose Exposures in Teletherapy Of Prostate Cancer Using a Rectal Balloon," Strahlentherapie und Onkologie, Vol. 183, No. 11, 2007, pp. 617-624. doi:10.1007/s00066-007-1736-8

[39] C. Vargas, A. Martinez, L. L. Kerstin, et al., "Dose-Volume Analysis of Predictors for Chronic Rectal Toxicity after Toxicity after Treatment of Prostate Cancer with Adaptive Image-Guided Radiotherapy," International 
Journal of Radiation Oncology, Biology, Physics, Vol. 62, No. 5, 2005, pp. 1297-1308.

doi:10.1016/j.ijrobp.2004.12.052

[40] F. Paulson, C. Belka, M. Alber, W. Budach and M. Bam- berg, "Intensity Modulated Radiotherapy IMRT," Onkologe, Vol. 9, No. 3, 2003, pp. 315-327.

doi:10.1007/s00761-003-0495-0 